\title{
The 2-Microlocal Canonical Form for a Class of Microdifferential Equations and Propagation of Singularities
}

By

Nobuyuki TOSE*

\section{§ 0. Introduction}

We study a class of microdifferential equations with involutory double characteristics.

In [8], the author dealt with a class of equation whose microlocal model is

$$
P_{0} u=\left(D_{1} D_{2}+(\text { lower })\right) u=0
$$

defined in a neighborhood of $\left(0, d z_{3}\right) \in T^{*} \mathbb{C}^{n}$. He employed the theory of 2-microlocalization developed by M. Kashiwara and $Y$. Laurent (See for example [2] and [6].) and showed that (0.1) is equivalent to $D_{1} u=0$ or $D_{2} u=0$ or $u=0$ as a 2 -microdifferential equation.

In this paper, we generalize the result of [8] mentioned above to a class of microdifferential equation of which microlocal canonical form is

$$
\left.P_{1} u=\left(D_{1}^{m_{1}} D_{2}^{m_{2}}+\text { (lower }\right)\right) u=0
$$

defined in a neighborhood of $\left(0, d z_{3}\right) \in T^{*} \mathbb{C}^{n}$. We assume that

$$
\begin{aligned}
& P_{1} \text { has Regular Singularities along } \\
& \Lambda=\left\{(z, \zeta d z) \in T^{*} \mathbb{C}^{n} ; \zeta_{1}=\zeta_{2}=0\right\} \\
& \text { in the sense of Kashiwara-Oshima [3]. }
\end{aligned}
$$

We prove that $(0.2)$ is equivalent to 


$$
\left\{\begin{array} { c } 
{ D _ { 1 } u _ { 1 } = 0 } \\
{ \vdots } \\
{ \mathrm { D } _ { 1 } \dot { u } _ { m _ { 1 } } = 0 }
\end{array} \quad \text { or } \quad \left\{\begin{array}{c}
D_{2} u_{1}=0 \\
\vdots \\
D_{2} \dot{u}_{m_{2}}=0
\end{array} \quad \text { or } u=0\right.\right.
$$

as a 2-microdifferential equation.

In hyperbolic case, we study the propagation of microlocal singularities of the solutions of the equation whose model is (0.2).

Now we give the plan of this paper.

In $\S 1$ we prepare some notation about microlocal analysis and 2microlocal analysis.

In $\S 2$ we announce the main theorem.

In $\S 3$ we give the proof of the main theorem. In the course of the proof, we give the 2-microlocal canonical form of the equation (0.2).

The author would like to express his gratitude to Prof. H. Komatsu for guidance and encouragement.

\section{§1. Preliminaries and Notation}

1.1. Let $X$ be an open subset of $\boldsymbol{C}^{n}$ and let $T^{*} X$ be its cotangent bundle. We identify the zero section of $T^{*} X$ with $X$. Let $z=$ $\left(z_{1}, \ldots, z_{n}\right)$ be a coordinate system of $X$. Then $(z, \zeta \cdot d z)$ denotes a point of $T^{*} X$ with $\zeta \in C^{n}$. $T^{*} X$ is endowed with the sheaf $\mathscr{E}_{X}$ [resp. $\left.\mathscr{E}_{X}^{\infty}\right]$ of microdifferential operators of finite order [resp. of infinite order] constructed in [S. K. K.].

Throughout section $1, \Lambda$ denotes the following homogeneous regular involutory submanifold of $T^{*} X\left(=T^{*} X \backslash X\right)$.

$$
\Lambda=\left\{(z, \zeta) \in \stackrel{\circ}{T}^{*} X, \zeta_{1}=\cdots=\zeta_{d}=0\right\} \quad(1 \leqq d<n) .
$$

We prepare some notation concerning the second microlocalization or 2-microlocalization developed by Y. Laurent [6].

$X$ can be identified with the diagonal set of $X \times X$. Thus we obtain a canonical injection $T^{*} X \cong T_{X}^{*}(X \times X) \rightarrow T^{*}(X \times X)$ which defines an injection $\Lambda \rightarrow \Lambda \times \Lambda$. We remark that $\Lambda \times \Lambda$ is endowed with a canonical foliation. By definition, $\tilde{\Lambda}$ denotes the union of bicharacteristics of $\Lambda \times \Lambda$ which pass through $\Lambda$. $T_{\Lambda}^{*} \tilde{\Lambda}$ has the canonical coordinate $\left(z, \zeta^{\prime \prime} d z^{\prime \prime}, z^{\prime *} d z^{\prime}\right)$ with $z \in X, z^{\prime}=\left(z_{1}, \ldots, z_{d}\right), z^{*}=\left(z_{1}^{*}, \ldots, z_{d}^{*}\right)$ $\in C^{d}, z^{\prime \prime}=\left(z_{d+1}, \ldots, z_{n}\right)$ and $\zeta^{\prime \prime}=\left(\zeta_{d+1}, \ldots, \zeta_{n}\right) \in C^{n-d}$. Here $\left(z, \zeta^{\prime \prime}\right)$ 
denotes a point of $\Lambda$. We identify the zero section of $T_{A}^{*} \tilde{\Lambda}$ with $\Lambda_{\text {。 }}$ Y. Laurent [6] defined the sheaf $\mathscr{E}_{\Lambda}^{2, \infty}$ on $T_{A}^{*} \tilde{\Lambda}$ called the sheaf of 2-microdifferential operators of infinite order. $\mathscr{E}_{A}^{2, \infty}$ is constructed using the coordinates $\left(z, \zeta^{\prime \prime}, z^{*}\right)$ as follows.

Definition 1.1 (Y. Laurent [6])。 Let $U$ be an open set of $T_{A}^{*} \tilde{\Lambda}$. A formal sum $\sum_{(i, j) \in Z^{2}} P_{i, j}\left(z, \zeta^{\prime \prime}, z^{*}\right)$ belongs to $\Gamma\left(U, \mathscr{E}_{\Lambda}^{2, \infty}\right)$ if and only if the following conditions (A), (B) are satisfied.

(A) $P_{i, j}\left(z, \zeta^{\prime \prime}, z^{*}\right)$ is holomorphic on $U$ and homogeneous of order $j$ with respect to $\left(\zeta^{\prime \prime}, z^{\prime *}\right)$ and homogeneous of order $i$ with respect to $z^{*}$.

(B) For any compact subset $K$ of $U$, there exists a positive number $C_{K}$ and for any positive number $\varepsilon$ and any compact subset $K$ of $U$, there exists a positive number $C_{\varepsilon, K}$ such that the following estimates $\left(B_{1}\right) \sim\left(B_{4}\right)$ are satisfied.

$\left(\mathrm{B}_{1}\right) \sup _{K}\left|P_{i, i+k}\right| \leqq C_{\varepsilon, K} \frac{\varepsilon^{i+k}}{i ! k !}(i, k \geqq 0)$.

$\left(\mathrm{B}_{2}\right) \sup _{K}\left|P_{i, i+k}\right| \leqq C_{\varepsilon, K}^{-k} \varepsilon^{i} \frac{(-k) !}{i !}\left(\begin{array}{l}i \geqq 0 \\ k<0\end{array}\right)$.

$\left(\mathrm{B}_{3}\right) \sup _{K}\left|P_{i, i+k}\right| \leqq \varepsilon^{k} C_{K}^{-i} \frac{(-i) !}{k !}\left(\begin{array}{l}i<0 \\ k \geqq 0\end{array}\right)$.

$\left(\mathrm{B}_{4}\right) \sup _{K}\left|P_{i, i+k}\right| \leqq C_{K}^{-i-k}(-i) !(-k) !(i, k<0)$ 。

$\mathscr{E}_{A}^{2, \infty}$ is a ring extension of $\pi^{-1} \mathscr{E} X \mid \infty$, where $\pi$ is the canonical projection $T_{\Lambda}^{*} \tilde{\Lambda} \rightarrow \Lambda$. In fact, we have

$$
\left.\mathscr{E}_{X}^{\infty}\right|_{\Lambda} \longrightarrow \mathscr{D}_{\Lambda}^{2 \infty}=\left.\mathscr{E}_{\Lambda}^{2, \infty}\right|_{\Lambda}
$$

Here (1.2) is injective.

Here we give the sheaf $\mathscr{E}_{A}^{2}(r, 1)$, which is a subring of $\mathscr{E}_{A}^{2, \infty}$.

Definition 1.2. For $\left(i_{0}, j_{0}\right) \in \mathbb{Z}^{2}$ and $r(r>1) \in \mathbb{Q} \cup\{\infty\}, P=\sum P_{i j}$ $\left(\in \mathscr{E}_{A}^{2, \infty}\right)$ belongs to $\mathscr{E}_{\Lambda}^{2(r, 1)}\left[i_{0}, j_{0}\right]$ if and only if

$$
\begin{aligned}
& S(P)=\left\{(i, j-i) \in \mathbb{Z}^{2} ; P_{i j} \neq 0\right\} \\
& \subset\left\{(i, j-i) \in \mathbb{Z}^{2} ; \frac{1}{r} i+(j-i) \leqq \frac{1}{r} i_{0}+\left(i_{0}-j_{0}\right),\right. \\
& \left.i+(j-i) \leqq i_{0}+\left(j_{0}-i_{0}\right)\right\} .
\end{aligned}
$$

We define $\mathscr{E}_{A}^{2}{ }_{A}^{(r, 1)}$ by 


$$
\mathscr{E}_{\Lambda}^{2,(r, 1)}=\bigcup_{(i, j) \in Z^{2}} \mathscr{E}_{\Lambda}^{2,(r, 1)}[i, j]
$$

Definition 1.3. If $P=\sum P_{i, j}$ is a section of $\mathscr{E}_{\Lambda}^{2(r, 1)}\left[i_{0}, j_{0}\right]$ and is not a section of $\mathscr{E}_{\dot{\Lambda}}^{2(r, 1)}[i, j]$ which is strictly smaller than $\mathscr{E}_{\dot{\Lambda}}^{2(r, 1)}\left[i_{0}, j_{0}\right]$, then we put

$$
\sigma_{\Lambda}^{(r, 1)}(P)=P_{i_{0}, j_{0}},
$$

which is called the principal symbol for $P$ along $\Lambda$ of type $(r, 1)$.

For details about 2-microdifferential operators, see Y. Laurent [6].

1.2. We prepare some notation about 2-microfunctions developed by M. Kashiwara-Y. Laurent.

Let $M$ be an open subset of $\boldsymbol{R}^{n}$ and $X$ be a complexification of $M$ in $\boldsymbol{C}^{n}$. $\quad M$ [resp. $X$ ] is equipped with a coordinate system $x=\left(x_{1}, \ldots, x_{n}\right)\left[\right.$ resp. $\left.z=\left(z_{1}, \ldots, z_{n}\right)\right]$. Then $(x, \sqrt{-1} \xi \cdot d x)$ denotes a point of $\sqrt{-1} T^{*} M\left(=T_{M}^{*} X\right)$ with $\xi=\left(\xi_{1}, \ldots, \xi_{n}\right) \in \mathbb{R}^{n} . \quad \sqrt{-1} T^{*} M$ is endowed with the sheaf $\mathscr{C}_{M}$ constructed in Sato-Kawai-Kashiwara [7].

Throughout section $1, \Lambda^{R}$ is the following homogeneous regular involutory submanifold in $\sqrt{-1} T^{*} M\left(=\sqrt{-1} T^{*} M \backslash M\right)$.

$$
\Lambda^{R}=\left\{(x, \sqrt{-1} \xi d x) ; \xi_{1}=\cdots=\xi_{d}=0\right\} \quad(1 \leqq d<n) .
$$

Then $\Lambda$ is a complexification of $\Lambda^{R}$ in $\dot{T}^{*} X . \Lambda$ is a regular involutory submanifold of $\dot{T}^{*} X$, thus $\Lambda$ has a canonical foliation. $\tilde{\Lambda}^{R}$ denotes the union of bicharacteristics of $\Lambda$ passing through $\Lambda^{R}$.

$\tilde{\Lambda}^{R}$ is equipped with the sheaf $\mathscr{C}_{\tilde{\Lambda}^{R}}$ of microfunctions with holomorphic parameters $\left(z_{1}, \ldots, z_{d}\right)$.

$T_{\Lambda^{R}}^{*} \tilde{\Lambda}^{R}$ has a canonical coordinate system $\left(x, \sqrt{-1} \xi^{\prime \prime} d x^{\prime \prime}, \sqrt{-1} x^{\prime *} d x^{\prime}\right)$ with $x \in M, x^{\prime}=\left(x_{1}, \ldots, x_{d}\right), x^{\prime *}=\left(x_{1}^{*}, \ldots, x_{d}^{*}\right) \in \boldsymbol{R}^{d}, x^{\prime \prime}=\left(x_{d+1}, \ldots, x_{n}\right)$ and $\xi^{\prime \prime}=\left(\xi_{d+1}, \ldots, \xi_{n}\right) \in \mathbb{R}^{n-d}$. Here $\left(x, \sqrt{-1} \xi^{\prime \prime} d x^{\prime \prime}\right)$ denotes a point of $\Lambda^{R}$. We remark that $T_{\Lambda^{*}}^{*} \tilde{\Lambda}$ is a natural complexification of $T_{\Lambda^{R}}^{*} \tilde{\Lambda}^{R}$.

We define several sheaves.

$$
\mathscr{A}^{2}{ }_{\Lambda^{R}}=\left.\mathscr{C}_{\tilde{\Lambda}^{R}}\right|_{\Lambda^{R}} \text {. }
$$$$
\mathscr{B}^{2}{ }_{\Lambda^{R}}=\mathscr{H}^{d}{ }_{\Lambda^{R}}\left(\mathscr{C}_{{ }^{k}}\right) \text { (the sheaf of 2-hyperfunction). }
$$$$
\mathscr{C}^{2}{ }_{\Lambda^{R}}=\mathscr{H}_{T^{d}}{ }_{\Lambda^{R}} \tilde{\Lambda}^{R} \quad\left(\pi^{-1} \mathscr{C}_{\tilde{\Lambda}^{R}}\right)^{a} \text { (the sheaf of 2-microfunctions). }
$$ 
Here $\pi$ is the comonoidal transformation

$$
\pi:\left(\tilde{\Lambda}^{R} \backslash \Lambda^{R}\right) \cup T_{\Lambda^{R}}^{*} \tilde{\Lambda}^{R} \longrightarrow \tilde{\Lambda}^{R}
$$

and $a$ is the antipodal map $a: T_{\Lambda^{R}}^{*} \tilde{\Lambda}^{R} \rightarrow T_{\Lambda^{R}}^{*} \tilde{\Lambda}^{R}$ (if $\mathscr{F}$ is a sheaf on $T_{\Lambda^{R}}^{*} \tilde{\Lambda}^{R}, \mathscr{F}^{a}$ denotes the inverse image of $\mathscr{F}$ by $a$ )。

We remark that $\mathscr{C}^{2}{ }_{\Lambda}^{R}$ is an $\mathscr{E}_{\Lambda^{2}}^{\infty}$ module.

We have the following fundamental exact sequences for $\mathscr{C}^{2}{ }_{1}^{R^{\circ}}$

$$
\begin{aligned}
& 0 \longrightarrow \mathscr{A}^{2}{ }_{\Lambda^{R}} \longrightarrow \mathscr{B}_{\Lambda^{R}}^{2} \longrightarrow \pi_{\Lambda^{R *}}\left(\mathscr{C}^{2}{ }_{\Lambda^{R}} \mid \dot{T}^{*}{ }_{\Lambda^{R}} \tilde{\Lambda}^{R}\right) \longrightarrow 0 \\
& \left.0 \longrightarrow \mathscr{C}_{M}\right|_{\Lambda^{R} \longrightarrow} \longrightarrow \mathscr{B}_{\Lambda^{R}}^{2} .
\end{aligned}
$$

Here

$$
\pi_{\Lambda^{R}}: \stackrel{\circ}{T}_{\Lambda^{R}}^{*} \tilde{\Lambda}^{R}\left(=T_{\Lambda^{R}}^{*} \tilde{\Lambda}^{R} \backslash \Lambda^{R}\right) \longrightarrow \Lambda^{R} .
$$

For details about the 2-microfunctions, see M. Kashiwara-Y. Laurent [2].

\section{\$2. Announcement of the Main Theorem}

We consider the following microdifferential equation defined on a neighborhood of $\dot{p} \in \sqrt{-1} T^{*} \mathbb{R}^{n}$ 。

$$
P u=\left(P_{1}^{m_{1}} P_{2}^{m_{2}}+Q\right) u=0 \text {. }
$$

Here we assume that

$$
\text { ord } P_{i}=k_{i} \quad(i=1,2)
$$

and that $Q$ is of strictly lower order than $P_{1}^{m_{1}}, P_{2}^{m_{2}}$. We put

$$
\left.\sigma\left(P_{i}\right)=p_{i} \text { (the principal symbol of } P_{i}\right) \text { 。 }
$$

We assume the following conditions $(2.4),(2.5),(2.6),(2.7)$ and (2.8).

$$
p_{1}(\not \circ)=p_{2}(\stackrel{p}{p})=0
$$

$$
p_{1}, p_{2} \text { are real. }
$$

$d p_{1}, d p_{2}$ and $\sqrt{-1} \sum_{i=1}^{n} \xi_{i} d x_{i}$ are linearly independent at $\stackrel{\circ}{\circ}$. $p_{1}^{-1}(0) \cap p_{2}^{-1}(0) \cap \sqrt{-1} T^{*} \mathbb{R}^{n}$ is involutory.

$P$ has Regular Singularities along $\Lambda=p_{1}^{-1}(0) \cap p_{2}^{-1}(0)$ in the sense of Kashiwara-Oshima [3]. 
In order to state the main theorem, we define the following regular involutory submanifold $\Lambda^{R}$ in $\sqrt{-1} T^{*} R^{n}$.

$$
\Lambda^{R}=p_{1}^{-1}(0) \cap p_{2}^{-1}(0) \cap \sqrt{-1} T^{*} \boldsymbol{R}^{n}
$$

The bicharacteristic of $\Lambda^{R}$ passing through $\not p$ is denoted by $\sum$.

Now we announce

Theorem 2.1. Let $u$ be a section of $\mathscr{C}_{\boldsymbol{R}^{n}}$ defined on a neighborhood of $\dot{p}$ that satisfies the equation (2.1). Then, there exist a neighborhood $U$ of $\dot{p}$ in $\sqrt{-1} T^{*} \boldsymbol{R}^{n}$ and a family $\left\{b_{t}^{1}\right\}_{t \in T_{1}}$ of integral curves on $\sum$ of $H_{p_{1}}$ and a family $\left\{b_{t}^{2}\right\}_{t \in T_{2}}$ of integral curves on $\sum$ of $H_{p_{2}}$, such that $\sum \subset s u p p u$ $\cap U$ is the union of $\cup_{t \in T_{1}} b_{t}^{1}$ and $\bigcup_{t \in T_{2}} b_{t}^{2}$ and some of connected components of $\left(\sum \cap U\right) \backslash\left(\bigcup_{t \in T_{1}} b_{t}^{1} \cup \bigcup_{t \in T_{2}} b_{t}^{2}\right)$

Remark 2. 2. T. Kobayashi ([5] and [4]) deals with the similar but more general class of differential equations with analytic coefficients. But his results are restricted to the case of differential equations and the method employed there is completely different from that in this paper.

Moreover the result in this paper about propagation of microlocal singularities is sharper.

By Kashiwara-Kawai [9], it is possible to prove that (2.10) if $u$ is a microfunction solution to (2.1) in Theorem 2. 1 and if

$$
\operatorname{supp} u \cap \sum \cap\left(\sum_{p}^{+} \backslash\{\not p o\}\right)=\emptyset
$$

then $\stackrel{\circ}{\text { }}$ supp $u$. Here

$$
\sum_{\not p}^{+}=\left\{\exp \left(s_{1} H_{p_{1}}\right) \circ \exp \left(s_{2} H_{p_{2}}\right)(\stackrel{\circ}{p}) ; s_{1}, s_{2} \geqq 0\right\}
$$

where $\exp \left(s_{i} H_{p_{i}}\right)(q)$ is the flow of $H_{p_{i}}$ issued from $q$.

As a corollary to Theorem 2.1, we can prove

Theorem 2.4. Let $u$ be a microfunction solution to (2.1) in Theorem 2. 1. If supp $u \cap U \cap \Sigma \cap\left(\Gamma^{1} \cup \Gamma^{2}\right)=\emptyset$, then $\not p \notin s u p p$ u. Here

$$
\Gamma^{i}=\left\{\exp \left(s_{i} H_{p_{i}}\right)(\stackrel{p}{p}) ; s_{i}>0\right\} \quad(i=1,2) .
$$




\section{§3. Proof of the Main Theorem 2-microlocal Canonical Form}

By Grigis-Lascar [1], it is sufficient to study the equation of the form

$$
P u=\left(D_{1}^{m_{1}} D_{2}^{m_{2}}+Q(x, D)\right) u=0
$$

when one wants to prove Theorem 2. 1. Here we assume that $P$ is a microdifferential operator defined in a neighborhood of

$$
q_{0}=\left(0, \sqrt{-1} d x_{3}\right) \in \sqrt{-1} T^{*} \mathbb{R}^{n}
$$

and that

$$
\text { ord } Q\left(x, D_{x}\right) \leqq m_{1}+m_{2}-1 \text {. }
$$

Moreover we assume that

$$
\begin{aligned}
& P \text { has Regular Singularities along } \\
& \Lambda=\left\{(z, \zeta d z) \in T^{*} C^{n} ; \zeta_{1}=\zeta_{2}=0\right\} \\
& \text { in the sense of Kashiwara-Oshima [3]. }
\end{aligned}
$$

We remark that the structure of the microdifferential equation (3.1) is well known outside $\Lambda$ after Sato-Kawai-Kashiwara [7].

We put for convenience

$$
\begin{aligned}
\Lambda^{R} & =\Lambda \cap \sqrt{-1} T^{*} \mathbb{R}^{n} \\
& =\left\{(x, \sqrt{-1} \xi d x) \in \sqrt{-1} T^{*} \mathbb{R}^{n} ; \xi_{1}=\xi_{2}=0\right\} 。
\end{aligned}
$$

We study the equation (3.1) 2 -microlocally along $\Lambda$ and give the canonical form for (3.1) as a 2-microdifferential equation defined in a neighborhood of $q_{1}=\left(0 ; \sqrt{-1} d x_{3} ; \sqrt{-1} d x_{2}\right)$ that is a point of $T_{\Lambda^{R}}^{*} \tilde{\Lambda}^{R}$.

We write $Q\left(x, D_{x}\right)$ in the form

$$
Q\left(x, D_{x}\right)=\sum_{j} Q_{j}\left(x, D_{x}\right)
$$

where $Q_{j}(z, \zeta)$ is the $j$-th order symbol of $Q\left(x, D_{x}\right)$. Next we develop $Q_{j}(z, \zeta)$ by partial Taylor series with respect to $\zeta_{1}$ and $\zeta_{2}$ as

$$
Q_{j}(z, \zeta)=\sum_{\alpha_{1}, \alpha_{2} \in Z_{+}} Q_{j}^{\alpha_{1}, \alpha_{2}}\left(z, \zeta^{\prime \prime}\right) \zeta_{1}^{\alpha_{1}} \zeta_{2}^{\alpha_{2}}
$$

with $\zeta^{\prime \prime}=\left(\zeta_{3}, \ldots, \zeta_{n}\right)$. Then the symbol series of $Q\left(x, D_{x}\right)$ is $\left\{Q_{i j}\left(z, \zeta^{\prime \prime}\right.\right.$, $\left.\left.z^{*}\right)\right\}$ as a 2 -microdifferential operator. Here 


$$
Q_{i j}\left(z, \zeta^{\prime \prime}, z^{\prime *}\right)=\sum_{\alpha_{1}+\alpha_{2}=i} Q_{j}^{\alpha_{1}, \alpha_{2}}\left(z, \zeta^{\prime \prime}\right) z_{1}^{* \alpha_{1}} z_{2}^{*^{\alpha_{2}}}
$$

The assumption (3.3) implies that

$$
\left\{(j, i) ; Q_{i j} \equiv 0\right\} \subset\left\{(j, i) ; i \geqq 0, j \leqq m_{1}+m_{2}-1, i \geqq j\right\} .
$$

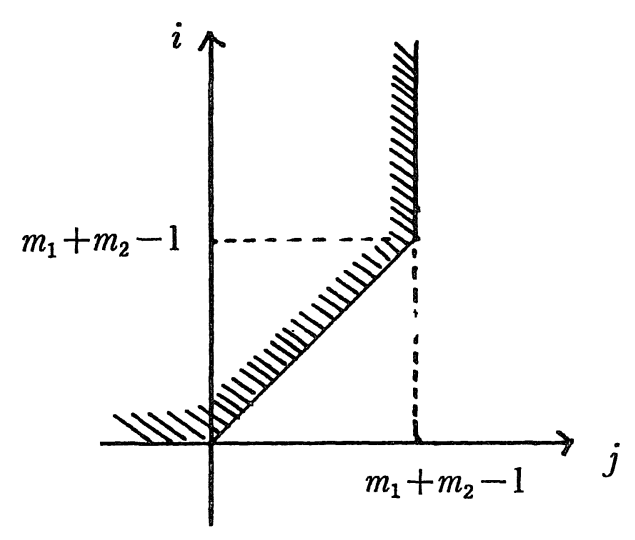

Figuse 3. 1

The right side of (3.7) is in Figure 3.1.

Because $D_{2}$ is invertible in $\mathscr{E}_{A}^{2(\infty, 1)}$ in a neighborhood of $q_{1}$, the equation (3.1) is 2 -microlocally equivalent to

$$
\left(D_{1}^{m_{1}}+R\left(x, D_{x}\right)\right) u=0 .
$$

Here

$$
R\left(x, D_{x}\right) \in \mathscr{E}_{\lambda}^{2(\infty, 1)}\left[m_{1}-1, m_{1}-1\right] .
$$

Hereafter we put $m=m_{1}$.

By Weierstrass type division theorem for $\mathscr{E}_{\Lambda}^{2(\infty, 1)}$ (see Laurent [6], Théorème 2.7.4), we may assume (3.8) is in the form

$$
\left(D_{1}^{m}+B_{0}\left(x, D^{\prime}\right) D_{1}^{m-1}+\cdots+B_{m-1}\left(x, D^{\prime}\right)\right) u=0
$$

with $D^{\prime}=\left(D_{2}, \ldots, D_{n}\right)$ and $B_{s}\left(x, D^{\prime}\right) \in \mathscr{E}_{\Lambda}^{2(\infty, 1)}[s, s]$.

Next we consider the equation (3.10) in the matrix form

$$
D_{1} U=M\left(x, D^{\prime}\right) U \text {. }
$$

Here $M\left(x, D_{x}\right)$ is a $m \times m$ matrix of 2-microdifferential operators and has a form 
(3. 12)

$$
M\left(x, D^{\prime}\right)=\left(\begin{array}{ccc}
01 & & \\
01 & & \\
\cdots & \\
\cdots & \\
& \cdots & \\
& & 0 \\
& & 01 \\
& & 01 \\
B_{m-1} & \cdots & B_{0}
\end{array}\right) .
$$

Theorem 3.1. In a neighborhood of $q_{1}=\left(0 ; \sqrt{-1} d x_{3} ; \sqrt{-1} d x_{2}\right)$, there exists $R\left(x, D^{\prime}\right) \in \mathscr{E}_{A}^{2, \infty} \otimes \operatorname{End}\left(\mathbb{C}^{m}\right)$ such that

$$
\left(D_{1} I_{(m)}-M\left(x, D^{\prime}\right)\right) R\left(x, D^{\prime}\right)=R\left(x, D^{\prime}\right)\left(D_{1} I_{(m)}\right)
$$

and that

$$
R\left(x, D^{\prime}\right) \text { is invertible. }
$$

Here $I_{(m)}$ denotes an identity matrix of degree $m$.

Thus

$$
\mathscr{E}_{A}^{2, \infty} / \mathscr{E}_{\Lambda}^{2, \infty} P \simeq\left(\mathscr{E}_{\Lambda}^{2, \infty} / \mathscr{E}_{A}^{2, \infty} D_{i}\right)^{m}
$$

Proof. We put

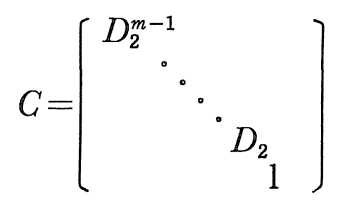

which is invertible in $\mathscr{E}_{A}^{2,(\infty, 1)}$.

We put

$$
\begin{aligned}
& F\left(x, D^{\prime}\right)=D_{1} I_{(m)}-C\left(D_{1} I_{(m)}-M\left(x, D^{\prime}\right)\right) C^{-1}
\end{aligned}
$$

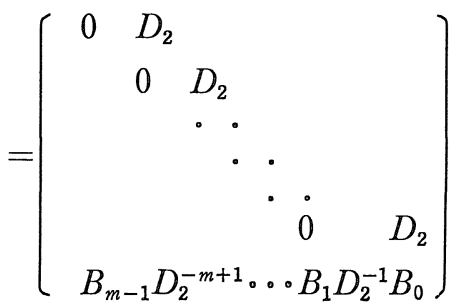

and 
(3. 18)

$$
N=\left[\begin{array}{lllll}
0 & D_{2} & & \\
& 0 & D_{2} & \\
& & \bullet & \bullet & \\
& & & \bullet & D_{2} \\
& & & & 0
\end{array}\right]
$$

We remark that

$$
\begin{cases}N^{l} \in \mathscr{E}_{\Lambda}^{2(\infty, 1)}[l, l] & (\text { for } 1 \leqq l \leqq m-1) \\ N^{l}=0 & (\text { for } l \geqq m) .\end{cases}
$$

We define $F_{0}\left(x, D^{\prime}\right)$ by

$$
F\left(x, D^{\prime}\right)=F_{0}\left(x, D^{\prime}\right)+N .
$$

Because $B_{s} D_{2}^{-s} \in \mathscr{E}_{\dot{\Lambda}}^{2(\infty, 1)}[0,0](s=0,1, \ldots, m-1)$, we have by $(3.18)$

$$
F\left(x_{1}^{(1)}, x^{\prime}, D^{\prime}\right) \cdots F\left(x_{1}^{(m)}, x^{\prime}, D^{\prime}\right) \in \mathscr{E}_{\Lambda}^{2 .(\infty, 1)}[m-1, m-1]
$$
with $x^{\prime}=\left(x_{2}, \ldots, x_{n}\right)$.

We construct $R\left(x, D^{\prime}\right) \in \mathscr{E}_{\Lambda}^{2, \infty} \otimes \operatorname{End}\left(\boldsymbol{C}^{m}\right)$ which satisfies

$$
\left(D_{1} I_{(m)}-F\left(x, D^{\prime}\right)\right) R\left(x, D^{\prime}\right)=R\left(x, D^{\prime}\right)\left(D_{1} I_{(m)}\right) \text {. }
$$

To obtain $R\left(x, D^{\prime}\right)$, we define $\left\{R^{(l)}\left(x, D^{\prime}\right)\right\}_{l \geqq 0}$ recursively by

$$
\left\{\begin{array}{l}
R^{(0)}\left(x, D^{\prime}\right)=I_{(m)} \\
\frac{\partial}{\partial x_{1}} R^{(l)}\left(x, D^{\prime}\right)=F\left(x, D^{\prime}\right) R^{(l-1)}\left(x, D^{\prime}\right) \quad(l=1,2, \ldots) .
\end{array}\right.
$$

In an explicit form, $R^{(l)}\left(x, D^{\prime}\right)$ is expressed as

$$
\begin{gathered}
R^{(l)}\left(x, D^{\prime}\right)=\int_{0}^{x_{1}} F\left(t^{(l)}, x^{\prime}, D^{\prime}\right) \int_{0}^{t^{(l)}} F\left(t^{(l-1)}, x^{\prime} D^{\prime}\right) \cdots \\
\cdots \int_{0}^{t^{(2)}} F\left(t^{(1)}, x^{\prime}, D^{\prime}\right) d t_{1} \cdots d t_{l} .
\end{gathered}
$$

By (3. 21),

$$
\begin{aligned}
& F\left(t^{(k)}, x^{\prime}, D^{\prime}\right) \cdots F\left(t^{(1)}, x^{\prime}, D^{\prime}\right) \\
& \quad \in \mathscr{E}_{\Lambda}^{2(\infty, 1)}[(k-m s)+s(m-1),(k-m s)+s(m-1)]
\end{aligned}
$$

where $s$ is the largest integer satisfying $m s \leqq k$.

We put formally

$$
R\left(x, D^{\prime}\right)=\sum_{l \geq 0} R^{(l)}\left(x, D^{\prime}\right)
$$

and prove $R\left(x, D^{\prime}\right)$ defines a section of $\mathscr{E}_{\Lambda}^{2, \infty}$. Then it is sufficient to show that $\sum_{l \geq 0} R^{(m l)}\left(x, D^{\prime}\right)$ is convergent in $\mathscr{E}_{\Lambda}^{2, \infty}$. To see it we define 
Definition 3.2 (Formal Norm). Let $V$ be an open set of $T_{\Lambda}^{*} \tilde{\Lambda}_{\text {。 }}$ For a compact set $K$ of $V$ and $P \in \Gamma\left(V, \mathscr{E}_{A}^{2,(\infty, 1)}[l, l]\right)$ we define a Formal Norm of $P$ on $K$ by

(3.27) $N_{K}^{[l, l]}(P, s, t)$ $=\sum \frac{2(2(n+2))^{j^{\prime}}\left(-j^{\prime}\right) !}{\left(-j^{\prime}+|\alpha|\right) !\left(-j^{\prime}+|\beta|\right) !} \sup _{K}\left|P_{l+i^{\prime} . l+j^{\prime}}^{\alpha, \beta}\right| s^{-2 i^{\prime}+\left|\alpha^{\prime}\right|+\left|\beta^{\prime}\right|} t^{-2 j^{\prime}+\left|\alpha^{\prime \prime}\right|+\left|\beta^{\prime \prime}\right|}$.

Here $\alpha=\left(\alpha^{\prime} ; \alpha^{\prime \prime}\right)=\left(\alpha_{1}, \alpha_{2} ; \alpha_{3}, \ldots, \alpha_{n}\right), \beta=\left(\beta^{\prime} ; \beta^{\prime \prime}\right)=\left(\beta_{1}, \beta_{2} ; \beta_{3}, \ldots, \beta_{n}\right) \in$ $\mathbb{Z}_{+}^{2} \times \mathbb{Z}_{+}^{n-2}$ and $P_{i, j}^{\alpha, \beta}=\left(\frac{\partial}{\partial z_{1}^{*}}\right)^{\alpha}\left(\frac{\partial}{\partial z_{2}^{*}}\right)^{\alpha}\left(\frac{\partial}{\partial \zeta^{\prime \prime}}\right)^{\alpha^{\prime \prime}}\left(\frac{\partial}{\partial z}\right)^{\beta} P_{i j 。}$

Remark 3.3. For $P_{s} \in \mathscr{E}_{\Lambda}^{2(\infty, 1)}\left[l_{s}, l_{s}\right](V) \quad(s=1,2)$, we have an inequality

$$
N_{K}^{\left[l_{1}+l_{2}, l_{1}+l_{2}\right]}\left(P_{1} P_{2}, s, t\right) \ll N_{K}^{\left[l_{1}, l_{1}\right]}\left(P_{1}, s, t\right) N_{K}^{\left[l_{2}, l_{2}\right]}\left(P_{2}, s, t\right) 。
$$

(Continue to the proof of Theorem 3.1.)

We define formally

$$
\sum R^{(m l)}\left(x, D^{\prime}\right)=\sum S_{i j}\left(x, D^{\prime}\right)
$$

where $S_{i j}$ is the symbol of order $[i, j]$. Then it is easy to see

$$
S_{i j}=\sum_{l(m-1) \geqq j} R_{i j}^{(m l)} \text {. }
$$

Here $R_{i j}^{(m l)}$ denotes the symbol of $R^{(m l)}$ with order $[i, j]$.

We take a compact neighborhood of $q_{1}$ and put

$$
\sup _{K \ni\left(z_{1}, \cdot\right)}\left|z_{1}\right|=h
$$

We have the estimates

$$
\begin{aligned}
& \sup _{K}\left|R_{i, i+k}^{(m l)}\right| \\
\leqq & \left(\frac{2(n+2)}{s}\right)^{(m-1) l} s^{2 i} t^{2 k} \frac{((m-1) l-i-k) !}{(2(n+2))^{i+k}} N_{K}^{[(m-1) l,(m-1) l]}\left(R^{(m l)}, s, t\right) \\
\leqq & s^{2 i} t^{2 k}\left(\left(\frac{2(n+2)}{s}\right)^{m-1} N_{K}^{[m-1, m-1]}\left(G_{1}, s, t\right)\right)^{l} \frac{h^{l}((m-1)-i-k) !}{(m l) !}
\end{aligned}
$$

where we put

$$
G_{1}=F\left(t^{(m)}, x^{\prime}, D^{\prime}\right) \cdots F\left(t^{(1)}, x^{\prime}, D^{\prime}\right) .
$$

We define a positive number $C_{2}$ by

$$
C_{2}=\left(\frac{2(n+2)}{s}\right)^{m-1} N_{K}^{[m-1, m-1]}\left(G_{1}, s, t\right) .
$$


Then by (3.32) we have

$$
\sup _{K}\left|S_{i, i+k}\right| \leqq \sum_{\substack{l(m-1) \geq i+k \\ l \geq 0}} C_{2}^{l} h^{l} \frac{((m-1) l-i-k) !}{(m l) !} .
$$

We define $\phi(i, k)$ by the right side of (3.34).

Estimates of $\phi(i, k)$ in case $i<0, k<0$.

$$
\begin{aligned}
& \phi(i, k) \\
& =\sum_{l \geqq 0} C_{2}^{l} h^{l} \frac{((m-1) l-i-k) !}{(m l) !} \\
& =\sum_{l \geqq 0} C_{2}^{l} h^{l} \frac{((m-1) l) !}{(m l) !} \cdot \frac{((m-1) l-i-k) !}{((m-1) l) !(-i) !(-k) !} \cdot(-i) !(-k) ! \\
& \leqq \sum_{l \geqq 0} C_{2}^{l} h^{l} 3^{(m-1) l-i-k}(-i) !(-k) ! \\
& =3^{(-i)} 3^{(-k)}(-i) !(-k) ! \sum_{l \geqq 0}\left(3^{m-1} C_{2} h\right)^{l} .
\end{aligned}
$$

By the estimates (3.35) above, if we take $h$ small enough and fix $(s, t)$ in (3.33) so that $N_{K}^{[m-1, m-1]}\left(G_{1}, s, t\right)$ is convergent, then we have a positive number $C_{K}$ such that

$$
\sup _{K}\left|S_{i, i+k}\right| \leqq(-i) !(-k) ! C_{K}^{-i-k} \text {. }
$$

Estimates of $\phi(i, k)$ in case $i \geqq 0, k<0$.

First we make a remark which appeared in Theorem 5.2.1 in Chapter 2 of Sato-Kawai-Kashiwara [7].

Remark 3. 4. If we take a positive number $C_{3}$ small enough, then for any positive number $\eta$ there exists a positive number $C_{\eta}$ such that

$$
\sum_{j \leqq(m-1) l} \frac{((m-1) l-j) !}{(m l) !} C_{3}^{l} \leqq \frac{\eta^{j} C_{\eta}}{j !}(j \geqq 0) .
$$

If we take a positive number $C_{3}$ small enough, there exists a positive number $C_{4}$ such that

$$
\sum_{l \geqq 0} \frac{((m-1) l-j) !}{(m l) !} C_{3}^{l} \leqq C_{4}^{(-j)}(-j) ! \quad(j<0) .
$$

In case that $i+k \geqq 0$, by (3. 37), for any positive number $\eta$ there exists a positive number $C_{\eta}$ such that

$$
\begin{aligned}
\phi(i, k) & \leqq s^{2 i} t^{2 k} C_{\eta} \frac{\eta^{i+k}}{(i+k) !} \\
& \leqq s^{2 i} t^{2 k} \frac{i !}{(i-(-k)) !(-k) !} \cdot \frac{(-k) !}{i !} \cdot C_{\eta} \eta^{i+k}
\end{aligned}
$$




$$
\leqq\left(2 s^{2} \eta\right)^{i}\left(\frac{1}{t^{2} \eta}\right)^{-k} C_{\eta} \frac{(-k) !}{i !}
$$

Thus, if we take $h$ in (3.33) small enough and fix $(s, t)$ in (3.33) so that $N_{K}\left(G_{1}, s, t\right)$ is convergent, then for any positive number $\varepsilon$ there exists a positive number $C_{\varepsilon, K}$ such that

$$
\sup _{K}\left|S_{i, i+k}\right| \leqq \varepsilon^{i} C_{\varepsilon, K^{-}}^{-k} \frac{(-k) !}{i !} \quad(i+k \geqq 0) .
$$

When $i+k<0$, we have a positive $G_{4}$ such that

$$
\begin{aligned}
\phi(i, k) & \leqq s^{2 i} t^{2 k} C_{4}^{-(i+k)}((-k)-i) ! \\
& \leqq s^{2 i} t^{2 k} C_{4}^{-i-k} \frac{((-k)-i) ! i !}{(-k) !} \circ \frac{(-k) !}{i !} \\
& \leqq s^{2 i} t^{2 k} C_{4}^{-i-k} \frac{(-k) !}{i !} \\
& \leqq\left(\frac{s^{2}}{C_{4}}\right)^{i}\left(\frac{C_{4}}{t^{2}}\right)^{-k} \frac{(-k) !}{i !}
\end{aligned}
$$

Because we can take $C_{4}$ as large as we like, there exists a positive number $C_{\varepsilon, K}$ for any positive number $\varepsilon$ such that

$$
\sup _{K}\left|S_{i, i+k}\right| \leqq C_{\varepsilon}^{(-k)} \varepsilon^{i} \frac{(-k) !}{i !} \quad(i+k<0) .
$$

After all, we have proved that $R\left(x, D^{\prime}\right)$ in $(3.26)$ defines a section of $\mathscr{E}_{A}^{2, \infty}$ in a neighborhood of $q_{1}$, which satisfies (3.21)。

Next we have to prove that $R\left(x, D^{\prime}\right)$ is invertible.

But we can prove it by applying the same argument of Theorem 5.2. 1 in Chapter 2 of Sato-Kawai-Kashiwara [7]. Q. E. D.

As a corollary to Theorem 3.1, we obtain a theorem about the propagation of 2-microlocal singularities for the solutions to (3.1).

We take a coordinate system of $T_{\Lambda^{R}}^{*} \tilde{\Lambda}^{R}$ as $\left(x, \sqrt{-1} \xi^{\prime \prime}, \sqrt{-1}\left(x_{1}^{*}, x_{2}^{*}\right)\right)$ with $\xi^{\prime \prime}=\left(\xi_{3}, \ldots, \xi_{n}\right)$ and define $\Sigma_{1}$ and $\Sigma_{2}$, subsets of $T_{\Lambda^{R}}^{*} \tilde{\Lambda}^{R}$ by

$$
\Sigma_{i}=\left\{\left(x, \sqrt{-1} \xi^{\prime \prime}, \sqrt{-1}\left(x_{1}^{*}, x_{2}^{*}\right)\right) ; x_{i}^{*}=0\right\} \quad(i=1,2) .
$$

Theoerm 3.5. Let $u$ be a section of $\mathscr{C}_{\Lambda^{R}}^{2}$ defined in a neighborhood of $q_{1}=\left(0 ; \sqrt{-1} d x_{3} ; \sqrt{-1} d x_{2}\right)$ [resp. $\left.q_{2}=\left(0 ; \sqrt{-1} d x_{3} ; \sqrt{-1} d x_{1}\right)\right]$ which satisfies (3.1). Then the support of $u$ in contained in $\Sigma_{1}\left[\right.$ resp. $\left.\Sigma_{2}\right]$. Moreover, 
the support of $u$ is the union of integral curves of $\partial / \partial x_{1}\left[r e s p . \partial / \partial x_{2}\right]$.

Proof. By Theorem 3.1, it is sufficient to show that the assertion holds for $u \in \mathscr{C}_{\Lambda^{R}}^{2}, q_{1}$ [resp. $u \in \mathscr{C}_{{ }^{R}}{ }^{R}, q_{2}$ ] satisfying $\partial / \partial x_{1} \cdot u=0$ [resp. $\left.\partial / \partial x_{2} \cdot u=0\right]$. But it is an easy consequence of de Rham's lemma for $\mathscr{C}_{\Lambda^{R}}{ }^{R}$ (See Appendix.)

From Theorem 3.5 above, we obtain

Theorem 3.6. Let $u$ be a microfunction defined in a neighborhood $\Omega$ of $q_{0}=\left(0, \sqrt{-1} d x_{3}\right)$ that satisfies (3.1) and $\Sigma$ be a bicharacteristic of $\Lambda^{R}$ that pass through $q_{0}=\left(0, \sqrt{-1} d x_{3}\right)$. Then there exists a family of integral curves on $\Sigma,\left\{b_{t}^{(1)}\right\}_{t \in T_{1}}$ of $\partial / \partial x_{1}$ and $\left\{b_{t}^{(2)}\right\}_{t \in T_{2}}$ of $\partial / \partial x_{2}$ such that supp $u \cap \Sigma$ is a union of $\cup_{t \in T_{1}} b_{t}^{(1)}, \cup_{t \in T_{2}} b_{t}^{(2)}$ and some of connected components of $(\Omega \cap \Sigma) \backslash\left(\underset{t}{\cup} b_{t}^{(1)} \cup \underset{t}{\cup} b_{t}^{(2)}\right)$.

Proof. We remark that there exists a canonical spectral map

$$
S p_{\Lambda^{R}}^{2}: \pi^{-1} \mathscr{B}_{\Lambda_{R}}^{2} \longrightarrow \mathscr{C}_{\Lambda^{R}}^{2}\left(\pi: T_{\Lambda^{R}}^{*} \tilde{\Lambda}^{R} \longrightarrow \Lambda^{R}\right)
$$

by Kashiwara-Laurent [2]. We put

$$
\gamma=S p_{\Lambda^{R}}^{2}(u) \cap\left(T_{\Lambda^{R}}^{*} \tilde{\Lambda}^{R} \backslash \Lambda^{R}\right) .
$$

Because $\gamma \subset \Sigma_{1} \cup \Sigma_{2}, \gamma$ is divided into two parts as

$$
\gamma=\left(\gamma \cap \Sigma_{1}\right) \cup\left(\gamma \cap \Sigma_{2}\right) .
$$

Moreover, $\gamma \subset \Sigma_{1}$ [resp. $\gamma \subset \Sigma_{2}$ ] is a union of integral curves of $\partial / \partial x_{1}$ [resp. $\left.\partial / \partial x_{2}\right]$ on $\Sigma_{1}\left[\right.$ resp. $\left.\Sigma_{2}\right]$. Thus, when we put $\Gamma_{i}=\pi\left(\gamma_{i}\right) \cap$ $\Sigma(i=1,2), \Gamma_{i}$ is written as

$$
\Gamma_{i}=\cup_{t \in T_{i}} b_{t}^{(i)}(i=1,2)
$$

by families of integral curves on $\Sigma:\left\{b_{t}^{(1)}\right\}_{t \in T_{1}}$ for $\partial / \partial x_{1}$ and $\left\{b_{t}^{(2)}\right\}_{t \in T_{2}}$ for $\partial / \partial x_{2}$.

By the fundamental exact sequences (1.10) and (1.11), we find that

$$
\Gamma_{1} \cup \Gamma_{2} \subset \operatorname{supp}(u) \cap \Sigma
$$

and that on $\Sigma \backslash\left(\Gamma_{1} \cup \Gamma_{2}\right) u$ has holomorphic parameters $\left(z_{1}, z_{2}\right)$ and 
thus has unique continuation property. (See Lemma 2.2.6 of Chapter 3 of Sato-Kawai-Kashiwara [7].) After all, we have proved the assertion of Theorem 3.6.

Q. E. D.

We have proved Theorem 2.1 by Theorem 3.6 above.

\section{Appendix. De $\mathbb{R}$ ham's Lemma for 2-Microfunctions}

We follow the notation prepared in the subsection $\S 1.2$ under the assumption $d \geqq 2$.

Let $\mathscr{M}$ be the coherent $\mathscr{D}_{X}$ module associated with

$$
\partial / \partial x_{i} \cdot u=0 \quad(1 \leqq i \leqq k<d) .
$$

We put $X_{0}=X \cap\left\{z_{1}=\cdots=z_{k}=0\right\}$ and $M_{0}=M \cap X_{0}$ and define projections $p: X \longrightarrow X_{0}$ and $p: M \longrightarrow M_{0}$ naturally. Then we have

$$
\mathbb{R} \mathscr{H}_{m \mathscr{D}_{X}}\left(\mathscr{M}, \mathcal{O}_{X}\right) \simeq p^{-1} \mathcal{O}_{X_{0}}
$$

We set $N=X \cap\left\{\operatorname{Im} z_{1}=\cdots=\operatorname{Im} z_{d}=0\right\}$ and $N_{0}=X_{0} \cap N$. We define a canonical projection $\tilde{\omega}: N \underset{N_{0}}{\times} T_{N_{0}}^{*} X_{0} \longrightarrow T_{N_{0}}^{*} X_{0}$. We identify $N \underset{N_{0}}{\times} T_{N_{0}}^{*} X_{0}$ with the submanifold of $T_{N}^{*} X$ and microlocalize (A.2) along $N$. Then we can derive an isomorphism

$$
\text { (A.3) } \quad \mathbb{R} \mathscr{H}_{\text {orse } \mathscr{E}_{X}}\left(\tilde{\mathscr{M}}, \mathscr{C}_{\tilde{A}^{R}}\right) \simeq \tilde{\omega}^{-1} \mathscr{C}_{\tilde{H}_{0}^{R}}
$$

by Lemma 2.2.3 of Chapter 1 of Sato-Kawai-Kashiwara [7]. Here $\widetilde{\mathscr{M}}$ is the associated $\mathscr{E}_{X}$ module for $\mathscr{M}$ and $\mathscr{C}_{\tilde{A}_{0}^{R}}$ denotes the sheaf on $\tilde{\Lambda}_{0}^{R}=T_{N_{0}}^{*} X_{0}$ of microfunctions with holomorphic parameters $\left(z_{k+1}, \ldots\right.$, $\left.z_{d}\right)$. We set $\Lambda_{0}^{R}=T_{N_{0}}^{*} X_{0} \cap T_{M_{0}}^{*} X_{0}$ and define a canonical projection $\phi: \Lambda^{R} \times T_{\Lambda_{0}^{R}}^{*}{ }_{\Lambda_{0}^{R}} \tilde{\Lambda}_{0}^{R} \longrightarrow T_{\Lambda_{0}^{R}}^{*} \tilde{\Lambda}_{0}^{R}$. We also identify $\Lambda^{R} \times T_{\Lambda_{0}^{R}}^{*} \Lambda_{0}^{R} \tilde{\Lambda}_{0}^{R}$ with the submanifold of $T_{\Lambda^{R}}^{*} \tilde{\Lambda}^{R}$. Microlocalize (A.3) along $\Lambda^{R}$, then we can deduce an isomorphism

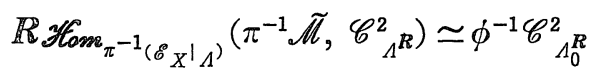

with $\pi: T_{\Lambda}^{*} \tilde{\Lambda} \rightarrow \Lambda$. Here $\mathscr{C}_{\Lambda_{0}^{R}}^{2}$ is the sheaf on $T_{\Lambda_{0}^{R}}^{*} \tilde{\Lambda}_{0}^{R}$ of 2 -microfunctions along $\Lambda_{0}^{R}$ 。 The above isomorphism is nothing but de Rham's lemma for 2-microfunctions. 


\section{References}

[1] Grigis A., Lascar R., Équationes locales d'un système de sous-variétés involutives, $C$. R. Acad. Sc. Paris, 283 (1976), 503-506.

[2] Kashiwara M. and Laurent Y, Théorèmes d'annulation et deuxième microlocalisations, Prépublication d'Orsay, Univ. Paris Sud, 1983.

[3] Kashiwara M. and Oshima T., Systems of microdifferential equations with regular singularities and their boundary value problems, Ann. of Math., 106 (1977), 145-200.

[4] Kobayashi T., On the singular Cauchy problem for operators with variable involutive characteristics, J. Fac. of Sci., Univ. of Tokyo, Sec. IA, 29 (1982), 97-142.

[5] - Elementary solutions and propagation of singularities for hyperbolic operators with involutive characteristics, Comm. in P. D. E., 7 (1982), 1189-1216.

[6] Laurent Y., Théorie de la deuxième microlocalisation dans le domaine complexe: opérateurs 2-microdifférentiels, Thesis presented to Univ. Paris-Sud, Centre d'Orsay, (1982): published by Birkhauser as Progress in Mathematics, 53, 1985.

[7] (referred to also as [S. K. K.]) Sato M., Kawai T. and Kashiwara M., Microfunctions and Pseudo-differential Equations, Lecture Note in Math., No. 287, Springer, (1973), 265529.

[8] Tose N., On a class of microdifferential equations with involutory characteristics, $J$. of Fac. Sci., Univ. Tokyo, 33-3 (1986) to appear.

[9] Kashiwara M. and Kawai T., Microhyperbolic pseudodifferential operators I, J. Math. Soc. Japan, 27-3 (1975), 359-404. 